\title{
Tri-Dirichlet-Type Problems with Polynomial Data in a Unit Sphere In $R^{3}$
}

\author{
Agah D. Garnadi
}

November 19, 2017

\begin{abstract}
We studied Tri-Dirichlet boundary value problem of TriLaplace equation. The problem is reformulated as a systems of Laplace-Poisson equation with Dirichlet problems. We utilize an exact algorithms for solving Laplace equations with Dirichlet conditions with polynomial functions data. The algorithm requires differentiation of the boundary function, but no integration.
\end{abstract}

\section{Introduction}

In this paper, we studied Bi-Laplace equation which have Tri-Dirichlet type boundary value problem with polynomials data in $R^{3}$. We approached the solution by Lemma that has been formulated by [2] and Lemma by [3].

Let $x=\left(x_{1}, x_{2}, x_{3}\right) \in R^{3}$, thus for $\alpha=\left(\alpha_{1}, \alpha_{2}, \alpha_{3}\right)$ of non-negative integers we say $x^{\alpha}$ as monomial $x_{1}^{\alpha_{1}} x_{2}^{\alpha_{2}} x_{3}^{\alpha_{3}}$. The degree of $x^{\alpha}$ is $\alpha_{1}+\alpha_{2}+\alpha_{3}$. A polynomial is said to be homogeneous of degree $m$ if it is a finite linear combination of monomial $x^{\alpha}$ of degree $m$ $; m=0,1,2, \ldots \ldots$. Let $\mathcal{P}_{m}$ denotes the vector space of polynomials in $R^{3}$, homogeneous of degree $m$, and $\mathcal{H}_{m}$ is the subspace of harmonic polynomials of degree $m$, then we have property by Axler and Ramey following

$$
\mathcal{P}_{m}=\mathcal{H}_{m}+|x|^{2} \mathcal{P}_{m-2}
$$

Theorem 1.1 if $p \in \mathcal{P}_{m}$, then $p=\Lambda_{m}(p)+|x|^{2} q$, for some $q \in \mathcal{P}_{m-2}$ and $\Lambda_{m}(p) \in \mathcal{H}_{m}$.

Theorem 1.1 leads to the following corollary, which gives the well-known decomposition (2) and an explicit formula for $p_{m}$.

Corollary 1.2 Every $p \in \mathcal{P}_{m}$ can be uniquely written in the form

$$
p=p_{m}+|x|^{2} p_{m-2}+\ldots+|x|^{2 k} p_{m-2 k},
$$

where $k=\left[\frac{m}{2}\right]$ and $p_{j} \in \mathcal{H}_{j}$ for each $j$. Furthermore, $p_{m}=\Lambda_{m}(0)$. 
Let $\Omega$ is a unit sphere in $R^{3}$, with the boundary $\Gamma=\partial \Omega=\left\{x \in R^{3} \mid \quad x_{1}^{2}+x_{2}^{2}+x_{3}^{2}<\right.$ $1\}$. The Tri-Dirichlet type problem can be formulated as following:

$$
\Delta^{2} u=0 \text { in } \Omega
$$

with boundary value problems

$$
\left.u\right|_{\Gamma}=p,\left.\Delta u\right|_{\Gamma}=q, \text { and }\left.\Delta^{2} u\right|_{\Gamma}=r,
$$

where, $p, q$, and $r$ is polynomials function.

The main result of this paper is to state the Theorem for the solution of problem in (2). The solution of this problem is a polynomial function that given in Theorem 3.1.

\section{Technical Lemmas}

This following Lemmas is needed to solve Tri-Dirichlet problem:

Lemma 2.1 If $p \in \mathcal{P}_{m}$, then the solution to the Dirichlet problem, $u \in \mathcal{P}_{m}$, with boundary data $\left.u\right|_{\Gamma}=p$ is

$$
p_{m}+p_{m-2}+\ldots+p_{m-2 k}
$$

where $k=\left[\frac{m}{2}\right]$ and $p_{m}, p_{m-2}, \ldots, p_{m-2 k}$ are the harmonic polynomials.

Proof:

Suppose $p \in \mathcal{P}_{m}$, because $|x|=1$ in boundary, by using (1) we have (3) equals to $p$ on $\Gamma$. Obviously (3) is harmonic on $R^{3}$, and hence its restriction to $\Omega$ is the solution to the Dirichlet problem.

Lemma 2.1 said that if we have polynomial data for dirichlet problem then we can obtain the solution of the Laplace equation with this boundary value problem. The solution is a linear combination of harmonic functions. We recommend you to study the algorithm to obtain this harmonic polynomials in [2].

By setting $p_{m-1}, p_{m-3}, \ldots$ are equal to 0 , the solution $u$ can be written as

$$
u=\sum_{j=0}^{m} p_{j}
$$

where $\left.\sum_{j=0}^{m} p_{j}\right|_{\Gamma}=p$.

Lemma 2.2 [Herzog, 2000] Given $p \in \mathcal{P}_{m}$ and $q \in \mathcal{P}_{m+2}$ then there exists $u \in \mathcal{P}_{m+2}$ such that

$$
\Delta u(x)=q(x) \quad x \in \Omega \quad \text { and }\left.\quad u\right|_{\Gamma}=p(x) .
$$

\section{Main Result}

Here we provide the Theorem about the solution of Tri-Dirichlet type problem. The Existence of this solution can be presented in the following Theorem: 
Theorem 3.1 Given $p \in \mathcal{P}_{m}$ and $q \in \mathcal{P}_{m+2}$ then there exists $u_{h} \in \mathcal{P}_{m}$ and $u_{2} \in \mathcal{P}_{m+2}$ such that $u=u_{h}+u_{p}$ is the solution of (2).

Proof: The Tri-Dirichlet type problem was presented in (2) can be writen as following:

$$
\Delta^{2} u=v \quad ;\left.u\right|_{\Gamma}=p,\left.\Delta u\right|_{\Gamma}=q,
$$

and

$$
\Delta v=f \quad ;\left.v\right|_{\Gamma}=r .
$$

The solution of problem (4) can be formulated if we had solved the problem (5). The solution of problem (5), $v \in \mathcal{P}_{m+2}$, can be obtained by using Lemma 2.1 which is a harmonic polynomials function.

Suppose that the solution of (5) is $v=v_{h}+v_{p}$, where $v_{h}$ is the solution of this problem:

$$
\Delta v_{h}=0 \quad ;\left.v_{h}\right|_{\Gamma}=p,
$$

and $u_{p}$ is the solution of this problem:

$$
\Delta v_{p}=f \quad ;\left.v_{p}\right|_{\Gamma}=0 .
$$

The solution $v_{h}$ can be obtained by using Lemma 2.1 which is the harmonic polynomials function. Next, by solving problem (7) when the solution of this problem is guaranteed by Lemma 2.2, so we have $v_{p}$.

\section{References}

[1] Sheldon Axler, Paul Bourdon, and Wade Ramey, Harmonic Function Theory, Graduate Text in Mathematics, Springer-Verlag, 1992.

[2] S. Axler and W. Ramey, Harmonic polynomials and Dirichlet type problems, proc. Amer. Math. Soc. 123(12) (1995), 3765-3773.

[3] G. Herzog, Polynomials solving Dirichlet boundary value problems, Amer. Math. Mothly 107(10) (2000), 934-936. 